\title{
The withdrawal of replacement therapy and outcomes in alpha-1 antitrypsin deficiency lung transplant recipients
}

\author{
To the Editor:
}

Alpha-1 antitrypsin deficiency (AATD) accounts for 5\% of lung transplants performed worldwide. Ireland has a high frequency of 1 in 25 for the $Z$ allele and 1 in 10 for the $S$ allele [1]. These variants are associated with decreased production of alpha-1 antitrypsin (AAT), which predisposes to unprotected proteolytic activity of neutrophil elastase (NE) and proteinase 3 (PR3), linked to an increased risk of emphysema and hepatic disease. Associated inflammatory conditions such as vasculitis, panniculitis and inflammatory bowel disease are also suspected to be caused by uncontrolled neutrophil proteinase activity [2-4]. Vascular problems, including aortic aneurysm, in AATD patients are more severe and may reflect abnormal elastic tissue properties potentially impacting on wound healing [5].

We observed that lung transplant recipients with AATD have suffered from specific complications, potentially related to the pathophysiology of their underlying disease. Therefore, we evaluated the incidence of complications and survival of recipients with AATD and a control group of recipients with chronic obstructive pulmonary disease (COPD) following lung transplantation.

We conducted a retrospective review of the data of all recipients with COPD and AATD transplanted at our centre. Data collection included demographic and functional baseline of recipients for both groups from May 2005 to December 2017. Genetic characteristics of the AATD group, the use of pre-transplant replacement therapy and time from listing to transplant were collected. Recipients were followed up from transplant until December 2018 or death. The primary end-point was the incidence of post-transplant complications. Any recipients with bronchial anastomotic complications (BAC) had bronchoalvelor lavage (BAL) sampling to determine total neutrophil count and a serum AAT level was performed. Secondary end-points included a comparison of the decline in forced expiratory volume in $1 \mathrm{~s}(\mathrm{FEV} 1)$ at year 1,5 and 10 from the time of transplant and survival between groups. Statistical analyses were performed with SPSS Statistics.

A total of 222 lung transplant recipients were studied. Of these, 42 (19\%) recipients had a diagnosis of either AATD or COPD: 14 AATD and 28 COPD. Baseline characteristics included a male preponderance of $71 \%$ in the AATD group versus $43 \%$ in the COPD group. AATD recipients were younger compared to those with COPD (AATD 60 \pm 9.2 years, COPD 64.9 \pm 5.5 years; $\mathrm{p}=0.04$ ). There was no significant difference in rates of double lung transplantation (AATD 79\%, COPD 54\%; $\mathrm{p}=0.18$ ) or the magnitude of post-transplant improvement in $\mathrm{FEV}_{1}(\mathrm{p}=0.98)$ between the groups. AATD phenotyping was $10 \mathrm{ZZ/Z0}$ (five ZZ and five Z0), two MZ and two patients' data were not available. Three AATD recipients had received replacement therapy with human alpha-1 proteinase inhibitor prior to transplant.

BAC were only seen in the AATD group who had received double lung transplant $(n=4 ; p=0.01)$. The median onset was 4 months after transplant. These four recipients were treated with endoscopic cryotherapy. Three required intervention with balloon bronchoplasty and two had bilateral stents inserted. Two recipients (Z0 mutation) with BAC suffered bronchial dehiscence and were listed for re-transplant due to failure to wean from mechanical ventilatory support. One of them subsequently underwent redo single lung transplant. Both recipients ultimately died from sepsis related to dehiscence and poor healing of bronchial anastomosis. Laboratory AAT levels in these four cases were low $0.21 \mathrm{~g} \cdot \mathrm{L}^{-1}(\mathrm{ZZ}), 0.83 \mathrm{~g} \cdot \mathrm{L}^{-1}$ (MZ), $0.42 \mathrm{~g} \cdot \mathrm{L}^{-1}(\mathrm{Z} 0)$ and $0.43 \mathrm{~g} \cdot \mathrm{L}^{-1}(\mathrm{Z} 0)$ (normal range $0.9-2.0 \mathrm{~g} \cdot \mathrm{L}^{-1}$ ). Total neutrophil count in BAL

@ERSpublications

Our study suggests an association between timing of withdrawal of replacement therapy and bronchial anastomotic complications after lung transplantation http://ow.ly/jhuP30o9dSS

Cite this article as: Kleinerova J, Ging P, Rutherford C, et al. The withdrawal of replacement therapy and outcomes in alpha-1 antitrypsin deficiency lung transplant recipients. Eur Respir J 2019; 53: 1900055 [https://doi.org/10.1183/13993003.00055-2019]. 
samples was high in both Z0 mutation cases affected by bronchial dehiscence, $90 \%$ and $85 \%$, versus $<10 \%$ in other two cases with BAC. AATD recipients without BAC had a mean bronchial neutrophil count of 19\% (95\% CI 2.6-35.4) 3 months after transplantation. There were no significant microbiological cultures identified in any AATD recipients at transplant or 3 months after transplant. Specifically, the two recipients with bronchial dehiscence had culture negative BAL 3 months post-transplant. Three of the four recipients with $\mathrm{BAC}$ had been receiving replacement therapy, which was discontinued at the time of listing for transplant. Replacement therapy in recipients with BAC (mutation one MZ, two Z0) was withdrawn 4.5 months prior to transplant (95\% CI -1.9-10.9). In the two recipients (mutation Z0) who died following bronchial dehiscence, replacement therapy had been withdrawn 1.3 months (95\% CI $0.8-1.7$ ) prior to transplant. In comparison, one AATD recipient with BAC (MZ mutation) who had replacement therapy discontinued 11 months prior to transplant and one AATD recipient with BAC (ZZ mutation) who did not receive replacement therapy had sustained survival of 5.5 years and 7.8 years, respectively. The other 10 AATD transplant recipients had never received replacement therapy.

Bowel inflammation associated with bowel ischaemia was observed in three AATD recipients versus none in the COPD group ( $\mathrm{p}=0.03$ ), causing death in two patients (both $\mathrm{ZZ}$ mutation). The median time to bowel ischaemia was 4.5 years after transplant. One patient (ZZ genotype) progressed to liver cirrhosis within 5 years of transplant. There was no significant difference in other common post-transplant complications (table 1).

The median survival was 10.9 years in the AATD group and 8.1 years in the COPD group ( $\mathrm{p}=0.3$ ). There was no statistical difference in FEV1 decline at year 1,5 and $10(p=0.31)$ between groups.

These results extend the available knowledge of post-transplant complications in AATD recipients observing a bimodal distribution of specific complications, first the early occurrence of bronchial anastomotic complications, and secondly late bowel complications. The complications described are potentially related to neutrophils as a source of NE and PR3, the driver of inflammation. The two recipients with the most severe BAC had BAL that confirmed high neutrophil activity. Once neutrophils are activated, they have an increased life span for the purpose of bacterial clearance [6]. It has been demonstrated that AATD lung transplant recipients show measurable free elastase activity in BAL fluid during severe lower respiratory tract inflammation, which was inhibited in ex vivo models by adding AAT [7]. We did not resume replacement therapy after transplant due to the limited evidence of efficacy in vitro in this setting [8]. Anastomotic complications with dehiscence were seen only in patients who had discontinued replacement therapy very shortly (a few weeks) before transplant. Our observation suggests a possible link between timing of withdrawal of replacement therapy and anastomotic complications. Increased neutrophil activity may occur as a rebound phenomenon following withdrawal of AAT replacement therapy. GULACK et al. [9] have also observed an increased incidence of dehiscence in AATD

\begin{tabular}{|c|c|c|c|c|}
\hline Post-transplant complication & AATD & COPD & $\begin{array}{l}\text { Outcome (death } \\
\text { AATD/COPD) }\end{array}$ & p-value \\
\hline Total recipients & 14 & 28 & & \\
\hline \multicolumn{5}{|l|}{ Associated with NE activity } \\
\hline Anastomotic complication & 4 (2 Z0, 1 ZZ, $1 \mathrm{MZ})$ & 0 & $2 / 0$ & 0.01 \\
\hline Other tissue fragility & $1(Z Z)$ & 1 & $1 / 0$ & 0.33 \\
\hline Bowel ischaemia & 3 (3 ZZ) & 0 & $2 / 0$ & 0.03 \\
\hline Liver steatosis pre-transplant & $3(Z Z)$ & 2 & $0 / 0$ & 0.31 \\
\hline Liver cirrhosis post-transplant & $1(\mathrm{ZZ})$ & 0 & $0 / 0$ & 0.33 \\
\hline \multicolumn{5}{|l|}{ Common complication } \\
\hline Acute rejection & 0 & 1 & $0 / 1$ & $>0.9$ \\
\hline CLAD & 0 & 4 & $0 / 1$ & 0.28 \\
\hline Sepsis & 1 (NA) & 4 & $1 / 4$ & 0.65 \\
\hline Lung cancer & $2(1 \mathrm{ZZ}, 1 \mathrm{NA})$ & 4 & $0 / 3$ & $>0.9$ \\
\hline PTLD & 0 & 1 & $0 / 1$ & $>0.9$ \\
\hline Other malignancy & $1(Z Z)$ & 1 & $0 / 0$ & $>0.9$ \\
\hline Heart failure & 0 & 1 & $0 / 1$ & $>0.9$ \\
\hline
\end{tabular}

Some patients had more than one complication but one primary cause of death was chosen. $p$-values in bold are statistically significant. AATD: alpha-1 antitrypsin deficiency; COPD: chronic obstructive pulmonary disease; NE: neutrophil elastase; CLAD: chronic lung allograft dysfunction; PTLD: post-transplant lymphoproliferative disorders. 
recipients, but data regarding augmentation before and during transplant were not available. Recently, the Hannover group described that pre-transplant replacement therapy was associated with worse survival after transplant, but no clear explanation for the reason of this finding was identified [10]. That study did not explore the impact of timing of withdrawal and did not identify AATD-specific complications. Our study suggests an association between withdrawal of replacement therapy and outcome. Despite the small number of subjects in this single centre retrospective analysis, our population was enriched with severe disease genotypes and a prolonged follow-up looking at disease specific complications.

Our study highlights a possible association between altered healing and withdrawal of replacement therapy. Further prospective multicentre studies are needed to examine this possible association and develop strategies to improve outcomes.

Jana Kleinerova, Patricia Ging, Colin Rutherford, Iain Lawrie, Sara Winward, Donna Eaton, Karen C. Redmond and Jim J. Egan

National Heart and Lung Transplant Centre, Mater Misericordiae University Hospital, Dublin, Ireland.

Correspondence: J. Kleinerova, Mater Misericordiae University Hospital, National Heart and Lung Transplant Centre, Eccles Street, Dublin, Ireland. E-mail: janakleinerova@hotmail.com

Received: Jan 082019 | Accepted: Feb 062019

Conflict of interest: None declared.

\section{References}

1 Carroll TP, O'Connor CA, Floyd O, et al. The prevalence of alpha-1 antitrypsin deficiency in Ireland. Respir Res 2011; 12: 91

2 Stone H, Pye A, Stockley RA. Disease associations in alpha-1-antitrypsin deficiency. Respir Med 2014; 108: 338-343.

3 Yang P, Tremaine WJ, Meyer RL, et al. $\alpha 1$-Antitrypsin deficiency and inflammatory bowel diseases. Mayo Clin Proc 2000; 75: 450-455.

4 Elzouki AN, Segelmark M, Wieslander J, et al. Strong link between the alpha 1-antitrypsin PiZ allele and Wegener's granulomatosis. J Intern Med 1994; 236: 543-548.

5 Vizzardi E, Corda L, Pezzali N, et al. Elastic properties of the ascending aorta in patients with $\alpha 1$-antitrypsin deficiency (Z homozygotes). Heart 2012; 98: 1354-1358.

6 Dunlea DM, Fee LT, McEnery T, et al. The impact of alpha-1 antitrypsin augmentation therapy on neutrophil-driven respiratory disease in deficient individuals. J Inflamm Res 2018; 11: 123-134.

7 King $\mathrm{MB}$, Campbell EJ, Gray $\mathrm{BH}$, et al. The proteinase-antiproteinase balance in alpha-1-proteinase inhibitor-deficient lung transplant recipients. Am J Respir Crit Care Med 1994; 149: 966-971.

8 Teschler $\mathrm{H}$. Long-term experience in the treatment of $\alpha 1$-antitrypsin deficiency: 25 years of augmentation therapy. Eur Respir Rev 2015; 24: 46-51.

9 Gulack BC, Mulvihill MS, Ganapathi AM, et al. Survival after lung transplantation in recipients with alpha-1-antitrypsin deficiency compared to other forms of chronic obstructive pulmonary disease: a national cohort study. Transpl Int 2018; 31: 45-55.

10 Conrad A, Janciauskiene S, Köhnlein T, et al. Impact of alpha 1-antitrypsin deficiency and prior augmentation therapy on patients' survival after lung transplantation. Eur Respir J 2017; 50: 1700962. 